\title{
On the Instability of Spheres Settling through a Vertical Pipe Filled with HPG
}

\author{
P. D. Weidman ${ }^{1 \dagger}$, B. Roberts ${ }^{2}$ and S. Eisen ${ }^{3}$ \\ ${ }^{1}$ Department of Mechanical Engineering, University of Colorado, Boulder, CO 80309-0427 \\ ${ }^{2}$ Control Solutions, Inc. 5560 N. Washington Street, Suite C6, Denver, CO 80216 \\ ${ }^{3}$ Boulder Wind Power, Inc., 2845 Wilderness Place, Suite 201, Boulder, CO 80301 \\ †Corresponding Author Email: weidman@colorado.edu
}

(Received July 30, 2011; accepted April 7, 2012)

\begin{abstract}
An extensive set of experiments were performed to determine the stability boundary for the longitudinal oscillation of steel spheres settling through Hydroxy Propyl Guar (HPG) contained in long vertical tubes, a phenomenon first reported by Mollinger, et al. (1999). Results are reported in d/D-pH parameter space, where $\mathrm{d}$ is the sphere diameter and $\mathrm{D}$ is the internal diameter of a tube. Longitudinal oscillations, which owe their existence to the self-healing nature of the gel, occur over the region $0.48<\mathrm{d} / \mathrm{D}<0.86$ and $8.31<\mathrm{pH}<8.82$. We discover that the upper $\mathrm{pH}$ limit of this stability domain is linked to the HPG gel point. During the course of experimentation, additional instabilities in the form of high-frequency transverse oscillations or helical motions were discovered and documented. The pure transverse oscillations found in the domain $8.035<\mathrm{pH}<8.31$ and $0.9<\mathrm{d} / \mathrm{D}<0.6$ result from the elastic nature of the guar in this region. For $6.25<\mathrm{pH}<8.035$ transverse oscillations or stable descents are interspersed between bands of tight helical motion at large $\mathrm{d} / \mathrm{D}$ and wide helical motion at low $\mathrm{d} / \mathrm{D}$. At the lowest value $\mathrm{pH}=6.25$ the guar is a Newtonian fluid, yet the spiral motion persists in small bands of d/D. We have verified, in the literature and through additional experiments using distilled water as the Newtonian liquid that the spiral motion of spheres falling inside vertical tubes does indeed exist.
\end{abstract}

\section{INTRODUCTION}

Preliminary observations of the vertical oscillation of steel spheres, periodically accelerating and decelerating while settling along the centerline of a non-Newtonian gel contained in vertical tubes, have been reported by Mollinger, et al. (1999). The fluid is formed from a Hydroxy Propyl Guar (HPG) gelling agent. The guar, a viscoelastic fluid that behaves as a self-healing gel, is commonly used for hydraulic fraction of oil reservoirs. Mollinger, et al. (1999) describes the longitudinal oscillations as follows:

The settling velocity is such that the fluid cannot relax all the stresses. The phenomenon can best be described by comparing the wake of the particle to a rubber band. When the rubber band is stretched too much it breaks, thereby enabling the particle to accelerate. Immediately a new rubber band starts to form slowing down the particle.

Mollinger, et al. (1999) presented their observations in tabular form listing the particle diameter $d$, pipe diameter $\mathrm{D}$, mean settling velocity, and the number $\mathrm{N}$ of downward jumps observed over the $1 \mathrm{~m}$ height of the pipe. Two pipes were used, one $24 \mathrm{~mm}$ in diameter and the other $30 \mathrm{~mm}$ in diameter. We have plotted their value of $\mathrm{N}$ versus d/D in Fig. 1. The solid line is a leastsquare fit to the data and the vertical dashed line is the perceived stability boundary for this HPG mixture. Mollinger, et al. (1999) did not report the $\mathrm{pH}$ of their mixture, but a detailed account of the preparation of the HPG from WG-11 powder obtained from Halliburton is given in their Appendix.

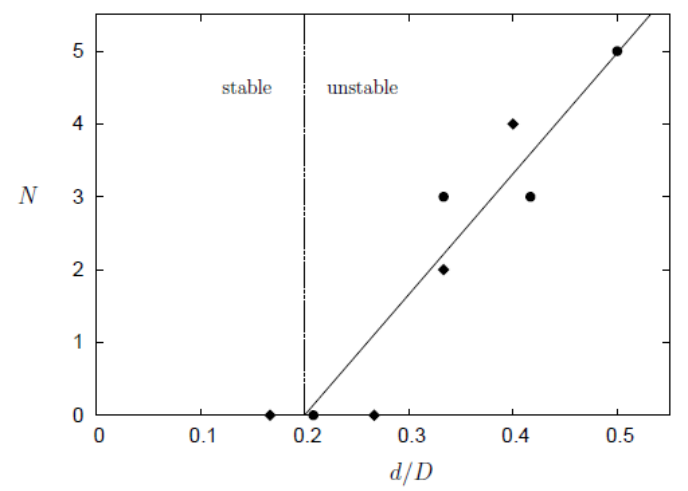

Fig. 1. Plot of the number $\mathrm{N}$ of jumps observed over an one meter sections of pipes versus the normalized sphere diameters d/D in the data of Mollinger, et al. (1999). The circles correspond to $\mathrm{D}=2.4 \mathrm{~cm}$ and the triangles correspond to $\mathrm{D}=3.0 \mathrm{~cm}$.

We undertook a repetition of the experiment reported in Mollinger, et al. (1999) to determine the entire region 
of longitudinal instability in a space of governing parameters. During this investigation we observed new instability modes in the HPG system. Preliminary results on our work have been reported at conference meetings but this is the first full account; see Roberts, et al. (2000), Weidman, et al. (2001) and Eisen, et al. (2002). The oscillatory motion of a solid sphere falling downward though a wormlike micellar fluid has been documented by Jayaraman \& Belmonte (2003) and Chen \& Rothstein (2004). A micellar fluid has viscoelastic properties, but it is not a guar. The oscillatory motion of cusped bubbles rising upward through micellar solutions has also been reported by Belmonte (2000) and Handzy \& Belmonte (2004). In these latter studies the length of a cusped bubble undergoes periodic oscillations as it ascends the micellar fluid.

\section{HYDROXY PROPYL GUAR AND ITS PREPARATION}

A gel is most simply defined as a class of fluid systems that shows solid-like properties in the presence of excess solvent (Lapasin \& Pricl, 1995). During gelation the fluid changes from one in which connectivity exists on only a very short-range scale to one in which the connectivity extends throughout the entire sample (Carnali, 1992). The test fluid used in this experiment is formed by titrating HPG with $\mathrm{NaOH}$ which has the effect of continuously altering the fluid properties from that of a Newtonian fluid at low $\mathrm{pH}$ to a semi-rigid gel at high $\mathrm{pH}$. In the present experiments we used WGA-1 hydroxylpropylguar gelling agent donated by Clearwater, Inc. The properties of HPG cross-linked with borate ions have been studied in great detail by Power, et al. (1998). They note that the HPG/borate system does not meet the criterion for being an ideal gel because the cross-links formed are not permanent, but continually break and reform. A characteristic feature of the HPG gel is that it has a high wall-slip tendency (Mollinger, et al. 1999).

The procedure for preparing the HPG used in our experiments follows closely that described by Power, et al. (1998). One slowly mixes 81 of distilled water (Safeway brand) with $0.36 \mathrm{~g} / \mathrm{l}$ boric acid and $0.48 \mathrm{wt} \%$ HPG gelling agent after which the liquid is stirred with a large stainless steel two-bladed impeller in an aluminum tank and heated to $60^{\circ} \mathrm{C}$ for three hours. Next the solution is slowly titrated with $10 \% \mathrm{NaOH}$ and is subsequently reheated to $60^{\circ} \mathrm{C}$ and stirred for an additional ten hours. Temperature control is maintained by a YSI Model 72 proportional controller and the heating is turned off automatically at ten hours. The guar is allowed to cool to room temperature while mixing another three to four hours. The HPG solution is then transferred to $2000 \mathrm{ml}$ beakers, poured into Erlenmeyer flasks and the pyrex tubes are fixed to the flasks through rubber stoppers (see $\$ 3$ for further details). The remainder of the HPG solution was poured into separate beakers and their $\mathrm{pH}$ was measured to two decimal places using an Orion 420A pH meter borrowed from our Chemical Engineering Department. The solutions were then separately poured to the rim of each pyrex tube in which the tests were made. The $\mathrm{pH}$ of each experiment was taken as the average of the $\mathrm{pH}$ values measured for each tube. Tiny bubbles escape the tubes in three to five hours, depending on the gel $\mathrm{pH}$. After experimentation, the glass tubes were cleaned with detergent and thoroughly rinsed with hot water. Hence, preparation for an experiment can take as long as 24 hours. The only deviation from the preparation method used by Power, et al. (1998) is that we did not transfer the solution to a set of rollers to facilitate blending; we simply continued stirring with our twobladed impeller. It should be noted that the HPG powder used by Powell, et al. (1998) was sourced from Dowell Schlumberger, while ours came from Clearwater, Inc.

Viscoelastic and gel properties of the HPG system are well documented in Power, et al. (1998). In particular, the values of the loss tangent $\tan (\delta)$ verus frequency obtained using a Weissenberg rheogoniometer are reported for values of $\mathrm{pH}$ in the range $6.25 \leq \mathrm{pH} \leq$ 11.29. The gel point for transition from liquid-like to solid-like behavior is defined as the point where the (frequency) gradient of the loss tangent curve changes from negative to positive. We plot their $\tan (\delta)$ gradient here in Fig. 2. In addition, Power, et al. (1998) provide extensive data on the linear viscoelastic rheology given by the dynamic storage modulus $\mathrm{G}^{\prime}$ (related to elasticity) and loss modulus $\mathrm{G}^{\prime \prime}$ (related to viscosity) as a function of $\mathrm{pH}$ for various rheogoniometer frequencies. A detailed discussion of these modulii and the method of their measurement may be found in Barnes, et al. (1989).

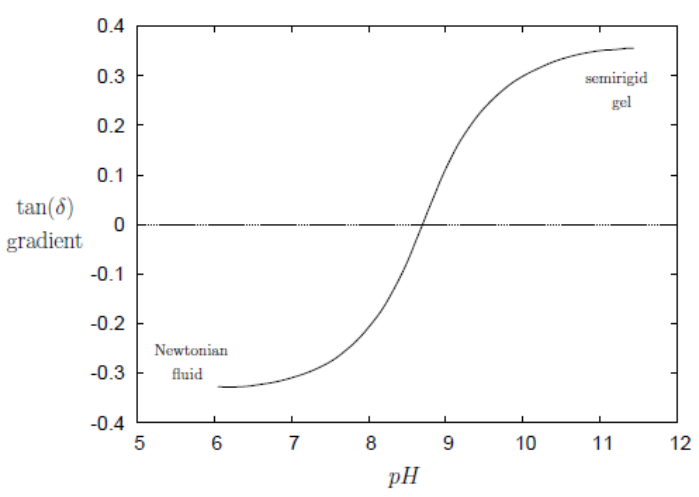

Fig. 2. Variation of the frequency gradient of $\tan (\delta)$ as a function $\mathrm{pH}$ showing the continuous behavior from a Newtonian fluid at low $\mathrm{pH}$ to a semi-rigid gel at high $\mathrm{pH}$. The value $\mathrm{pH}=8.7$ at which $\tan (\delta)=0$ gives the gel transition point. Adapted from Power, et al. (1989)

Power, et al. (1998) note that the $\mathrm{pH}$ for a solution of $0.36 \mathrm{~g} / \mathrm{l}$ boric acid and $0.48 \mathrm{wt} \% \mathrm{HPG}$ is approximately 6.2 at $20^{\circ} \mathrm{C}$, which is in the range $19-22^{\circ} \mathrm{C}$ of our laboratory experiments. The concentration of borate ions at this $\mathrm{pH}$ is negligible so the polymer solution exhibits no sign of cross-linking; hence the rheological behavior at $\mathrm{pH}=6.2$ is identical to that for an HPG solution for which no boric acid is present, i.e. it is a Newtonian fluid as depicted in Fig. 2.

\section{EXPERIMENTAL SETUP}

We denote $d$ the sphere diameter and $\mathrm{D}$ the internal diameter of the glass tubes. Two pyrex tubes $2.3 \mathrm{~m}$ in length, with diameters $D_{1}=2.596 \mathrm{~cm}$ and $D_{2}=3.033$ 
$\mathrm{cm}$, were constructed with millimeter graduations pasted over their entire length. These long tubes were obtained by fusing together two $1.15 \mathrm{~m}$ tubes aligned straight with a support fixture. The constructed tubes were mounted vertically side-by-side in front of a frosted plexiglass plate illuminated from behind with fluorescent lighting. The lower ends of the tubes were fitted through rubber stoppers into $2000 \mathrm{ml}$ Erlenmeyer flasks to collect the spheres. The upper ends of the tubes were fitted with rectangular plastic receptacles. Twenty precision steel spheres with diameters $1 / 16$ in < $\mathrm{d}<11 / 8$ in $(0.159 \mathrm{~cm}<\mathrm{d}<2.858 \mathrm{~cm})$ allowed testing for 36 different diameter ratios in the range $0.0523<$ $\mathrm{d} / \mathrm{D}<0.9785$; see Table 1 . The tubes were overfilled so that the plastic receptacles attached at the top contained about $2 \mathrm{~cm}$ of the guar used to immerse the test spheres and brush them free of bubbles prior to dropping. A test sphere was then picked up with a pair of tweezers and released along the centerline of the tube. For these slowly falling spheres, the motion could be closely tracked using a CCD camera mounted on a platform guided by vertical parallel rails and driven by a d.c. motor and cable system.

Table 1 Dimension $d$ of the steel spheres and the values of $d / D$ obtained using tubes for inner diameters $D_{1}=1.022$ and $D_{2}=1.200 \mathrm{in}$

\begin{tabular}{|c|c|c|c|}
\hline No. & d (in) & Tube & $d / D$ \\
\hline \hline 1 & $1 / 16$ & $D_{2}$ & 0.0523 \\
\hline 2 & $1 / 16$ & $D_{1}$ & 0.0612 \\
\hline 3 & $1 / 8$ & $D_{2}$ & 0.1047 \\
\hline 4 & $1 / 8$ & $D_{1}$ & 0.1223 \\
\hline 5 & $3 / 16$ & $D_{2}$ & 0.1570 \\
\hline 6 & $3 / 16$ & $D_{1}$ & 0.1835 \\
\hline 7 & $1 / 4$ & $D_{2}$ & 0.2094 \\
\hline 8 & $1 / 4$ & $D_{1}$ & 0.2446 \\
\hline 9 & $5 / 16$ & $D_{2}$ & 0.2617 \\
\hline 10 & $5 / 16$ & $D_{1}$ & 0.3058 \\
\hline 11 & $3 / 8$ & $D_{2}$ & 0.3141 \\
\hline 12 & $7 / 16$ & $D_{2}$ & 0.3664 \\
\hline 13 & $3 / 8$ & $D_{1}$ & 0.3669 \\
\hline 14 & $1 / 2$ & $D_{2}$ & 0.4188 \\
\hline 15 & $7 / 16$ & $D_{1}$ & 0.4281 \\
\hline 16 & $9 / 16$ & $D_{2}$ & 0.4711 \\
\hline 17 & $1 / 2$ & $D_{1}$ & 0.4892 \\
\hline 18 & $5 / 8$ & $D_{2}$ & 0.5235 \\
\hline 19 & $9 / 16$ & $D_{1}$ & 0.5504 \\
\hline 20 & $11 / 16$ & $D_{2}$ & 0.5760 \\
\hline 21 & $5 / 8$ & $D_{1}$ & 0.6115 \\
\hline 22 & $3 / 4$ & $D_{2}$ & 0.6281 \\
\hline 23 & $25 / 32$ & $D_{2}$ & 0.6543 \\
\hline 24 & $11 / 16$ & $D_{1}$ & 0.6727 \\
\hline 25 & $13 / 16$ & $D_{2}$ & 0.6805 \\
\hline 26 & $7 / 8$ & $D_{2}$ & 0.7328 \\
\hline 27 & $3 / 4$ & $D_{1}$ & 0.7339 \\
\hline 28 & $25 / 32$ & $D_{1}$ & 0.7644 \\
\hline 29 & $15 / 16$ & $D_{2}$ & 0.7852 \\
\hline 30 & $13 / 16$ & $D_{1}$ & 0.7950 \\
\hline 31 & 1 & $D_{2}$ & 0.8375 \\
\hline 32 & $7 / 8$ & $D_{1}$ & 0.8562 \\
\hline 33 & $17 / 16$ & $D_{2}$ & 0.8899 \\
\hline 34 & $15 / 16$ & $D_{1}$ & 0.9173 \\
\hline 35 & $9 / 8$ & $D_{2}$ & 0.9422 \\
\hline 36 & 1 & $D_{1}$ & 0.9785 \\
\hline & & & \\
\hline
\end{tabular}

The density, the storage modulus, and the loss modulus of the fluid mixture change with $\mathrm{pH}$, as does the time constant for self-healing of the gel after being subjected to shear forces. In addition to being dependent on mixture $\mathrm{pH}$, the sphere Reynolds numbers are a function of both the sphere diameter $\mathrm{d}$ and of the sphere/pipe diameter ratio $\mathrm{d} / \mathrm{D}$ due to blockage effects. This may be seen in the data presented in Fig. 3 obtained by dropping spheres through each tube and measuring their average descent rate over the central two meter sections of the tubes filled with an HPG mixture at $\mathrm{pH}=8.63$. Plotted is the average Reynolds number $\operatorname{Re}=\mathrm{Vd} / \mathrm{v}$ as a function of $\mathrm{d} / \mathrm{D}$ for each tube, where $\mathrm{V}$ is the average descent velocity and $v=3.06$ $\mathrm{cm} 2 / \mathrm{s}$ is the kinematic viscosity of the mixture measured using a Model 350 Cannon-Fensk viscometer. We realize that this is not the most accurate method for measuring the viscosity of this nonNewtonian liquid, but the qualitative difference in the Reynolds numbers for each tube is still readily apparent. In particular, at each diameter ratio $d / D$, the average Reynolds number in the smaller $D_{1}$ tube is about half that in the larger $D_{2}$ tube. Thus, at a minimum, we expect the longitudinal oscillations observed by Mollinger, et al. (1999) to be a function of $\mathrm{pH}, \mathrm{Re}$ and d/D. In our measurements, we found only a very small Reynolds number effect on the observed stability boundaries. For this reason, we simply report our findings in $\mathrm{pH}-\mathrm{d} / \mathrm{D}$ parameter space. It should be clearly pointed out, as mentioned above, that many rheological properties of the non-Newtonian liquid change with $\mathrm{pH}$.

We note in advance that our sample of the WGA-1 gelling agent was exhausted after about 15 preparations so another sample from Clearwater, Inc. was requested. At this point we ran into a consistency problem, since a given preparation using the new sample did not exactly match the $\mathrm{pH}$ obtained from the original sample. We then learned from Mollinger (private communication) that this was to be expected since the properties of samples obtained from different batches do not produce uniform results. About the same time we found that the borrowed $\mathrm{pH}$ meter was not working properly so we bought a new Orion 420A pH meter. During the experiments carried out over a two year period we made 53 preparations of the HPG but, owing to the aforementioned difficulties, we rejected the first ten runs as being unreliable. Of the remaining 43 preparations, a couple was rejected as being improperly mixed and others were used twice at the same approximate $\mathrm{pH}$ to double check stability boundaries and/or frequencies of oscillation. Mollinger advised us to allow sufficient time for the HPG mixture to recuperate after dropping a sphere because, especially at high $\mathrm{pH}$, the gel needs time to heal since dropping a sphere creates a hole in the fluid through which a second sphere can fall more easily. As a result we alternated our drops between tubes, so at least $15 \mathrm{~min}$ transpired between drops in a given tube. Finally, we note that a third sample of the WGA-1 gelling agent was obtained from Clearwater, Inc. in order to complete the experiments reported here. 


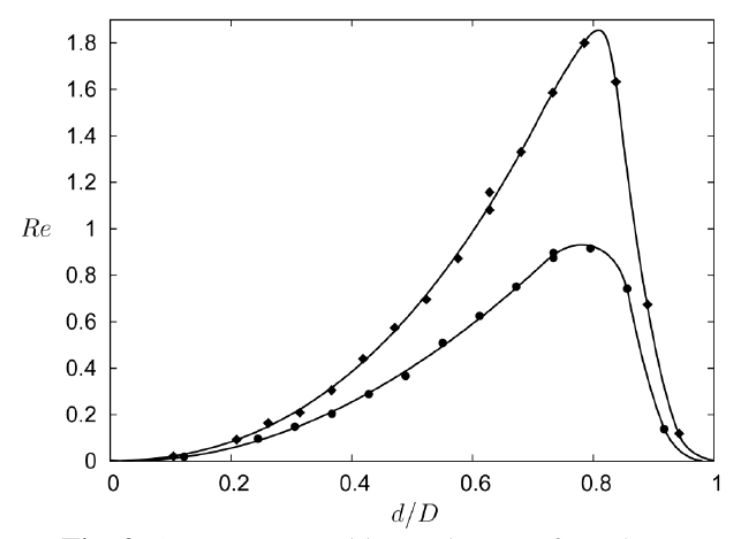

Fig. 3. Average Reynolds numbers Re for spheres falling down the two tubes filled with HPG at $\mathrm{pH}=$ 8:63. Circles are data from tube $D_{1}$ and triangles are data from tube $D_{2}$

\section{LONGITUDinAL OSCILLATIONS}

Our first experiments conducted near $\mathrm{pH}=8.60$ quickly revealed the longitudinal oscillations of spheres falling through the HPG. The spheres were consistently released by hand with tweezers on the axis of the tubes. All but the smaller spheres would immediately veer towards and slip down the tube wall, with or without longitudinal oscillation. Features of the slip motion was clearly observed by marking the spheres with black circles around orthogonal meridional and equatorial planes; no substantial rolling was evident in these longitudinal oscillations, except for little disturbances that occurred at mid-height where two glass tubes were joined together. For future reference, we define roll as the angular motion a sphere would have if rolling down against the tube wall and spin as the angular motion of a sphere about its vertical axis. In a few instances the longitudinal oscillations took place with the sphere descending along the tube axis. When this rare event did occur it was at a low value of d/D.

Our initial thought was to maintain axisymmetric flow in the system by drilling tiny holes through the spheres and release them on a fine wire aligned and drawn taut along the axis of a tube. However, the additional drag incurred by the sphere moving down the wire, with small annular clearance, inhibited the longitudinal oscillations. Hence it was decided to perform the experiments by releasing the spheres with tweezers along the tube axis and let them fall at will. A literature survey shows that the observed wall attraction for spheres and other bodies falling through viscoelastic fluids is a well known phenomenon; see for example, Dhahir \& Walters (1989) and Joseph, et al. (1992).

Space-time $(x-t)$ trajectories of the sphere motion were obtained using the CCD camera; here $\mathrm{x}$ is taken to be the downward coordinate and $t$ is time. Results at three values of $d / D$ for spheres sedimenting through HPG at $\mathrm{pH}=8.60$ are given in Fig. 4. For the lowest curve at $\mathrm{d} / \mathrm{D}=0.419$ no oscillations were observed in the length of our facility as indicated by the straight line trajectory. At $\mathrm{d} / \mathrm{D}=0.524$ a long period oscillation appears with amplitude $\Delta \mathrm{x}$ as defined in the figure, and at $\mathrm{d} / \mathrm{D}=0.628$ the oscillation period decreases while its amplitude increases. A plot of the average amplitude $\Delta \mathrm{x}$ taken from video data at several values of $d / D$ for an HGP mixture at $\mathrm{pH}=8.63$ is given in Fig. 5. Though, there is considerable scatter, this data is reminiscent of an Andronov-Hopf bifurcation. Thus, it have fitted the data to a Hopf curve of the form

$\Delta x=k \sqrt{\frac{d}{D}-\left(\frac{d}{D}\right)_{0}} \quad k=16.606,\left(\frac{d}{D}\right)_{0}=0.405$

shown as the sold line in Fig. 5.

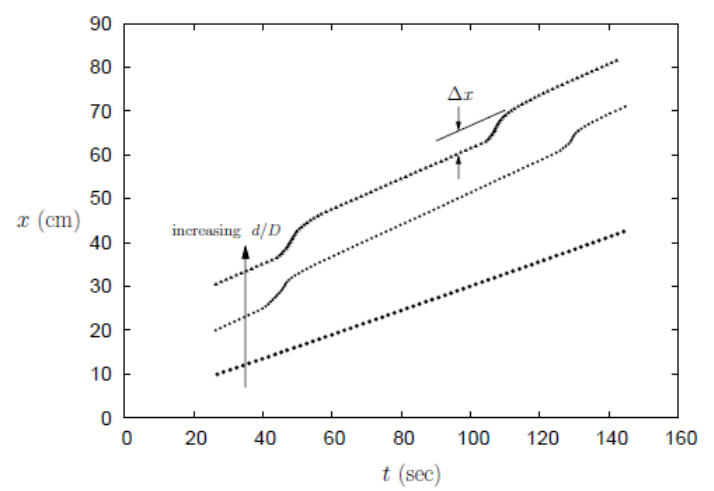

Fig. 4. Space-time trajectories for longitudinally oscillating spheres at $\mathrm{pH}=8.60$ in the large tube. The diamonds are for $\mathrm{d} / \mathrm{D}=0.419$; the circles are for $\mathrm{d} / \mathrm{D}=$

0.524 , and the triangles are for $\mathrm{d} / \mathrm{D}=0.628$

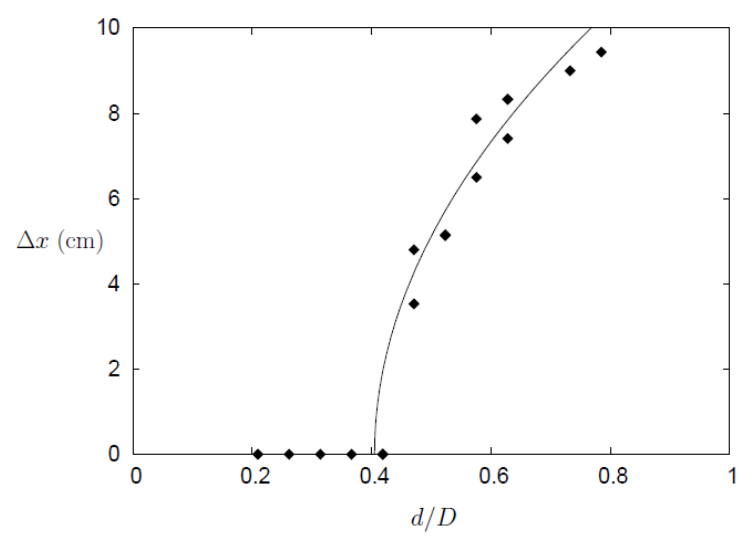

Fig. 5. Plot of the average amplitudes $\Delta x$ versus $d / D$ obtained from trajectory data recorded at $\mathrm{pH}=8.63$ in the large tube. All data are taken from tube $D_{1}$ and the solid line is a fitted Hopf bifurcation curve

The longitudinal oscillation frequencies, obtained by measurement of three to six oscillations with a stopwatch, appears to vary linearly with $\mathrm{d} / \mathrm{D}$ as shown in Fig. 6 for experiments taken at $\mathrm{pH}=8.43,8.52,8.77$ and 8.82. It may be inferred from Fig. 1 that Mollinder, et al. (1999) also noted an approximate linear variation of frequency with d/D. In Fig. 6 the solid circles/triangles represent data from the small/large tube. Unit Reynolds numbers effects are not apparent except at $\mathrm{pH}=8.52$ where the oscillation frequency in the smaller tube falls consistently below those in the larger tube. In each case oscillations are observed only over an intermediate range of $\mathrm{d} / \mathrm{D}$, the regions above and below being stable. Outside of the region of instability the descent speeds are attenuated and hence the gel is not so strongly sheared. For $\mathrm{d} / \mathrm{D} \rightarrow 0$ the attenuation in Reynolds numbers is linked to the reduced sphere diameters, while for $d / D \rightarrow 1$ the attenuation is the result of blockage effects; see Fig. 3. 
Also note that while the $\mathrm{d} / \mathrm{D}$ window of instability varies only slightly with $\mathrm{pH}$ in the first three panels of
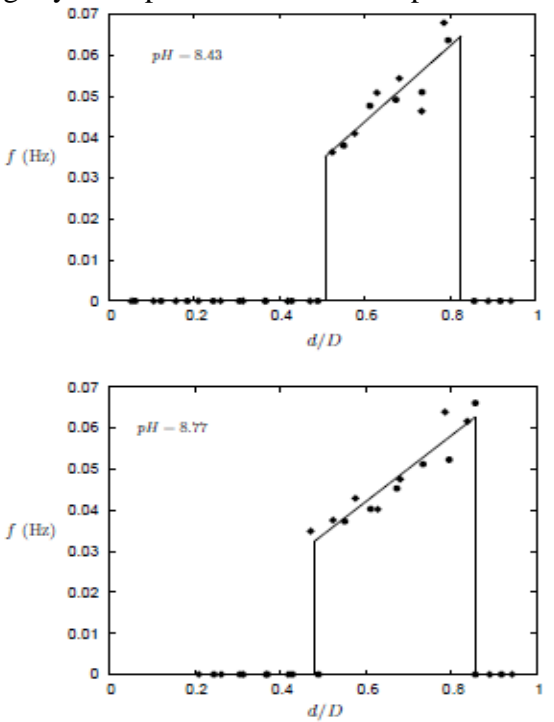

Fig. 6, that is a dramatic reduction in the instability window at $\mathrm{pH}=8.82$.
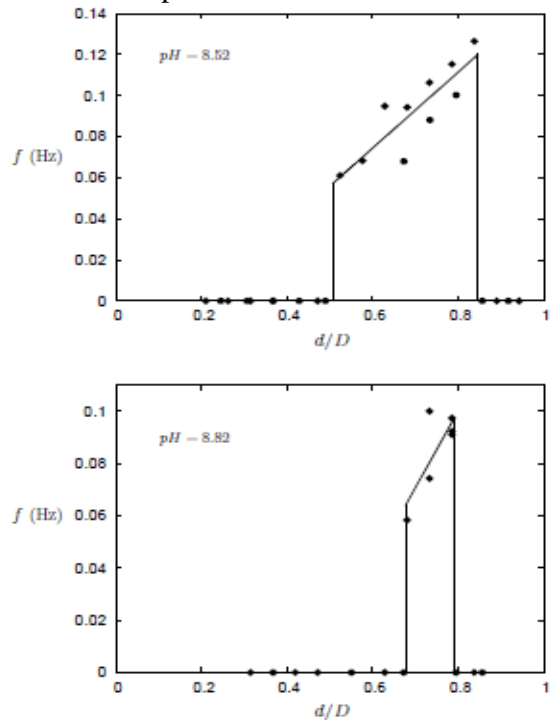

Fig. 6. Longitudinal oscillation frequencies as a function $\mathrm{d} / \mathrm{D}$ measured at different $\mathrm{pH}$. Circles are data from tube $D_{1}$ and triangles are data from tube $D_{2}$

This reduction in the $\mathrm{d} / \mathrm{D}$ window for longitudinal oscillations at $\mathrm{pH}=8.82$ is a signature of the closing of the stability boundary at its high $\mathrm{pH}$ limit as shown in Fig. 7. The open symbol on the left and the vertical dashed line on the right correspond to further unexpected phenomena we have observed which are discussed in the following sections.

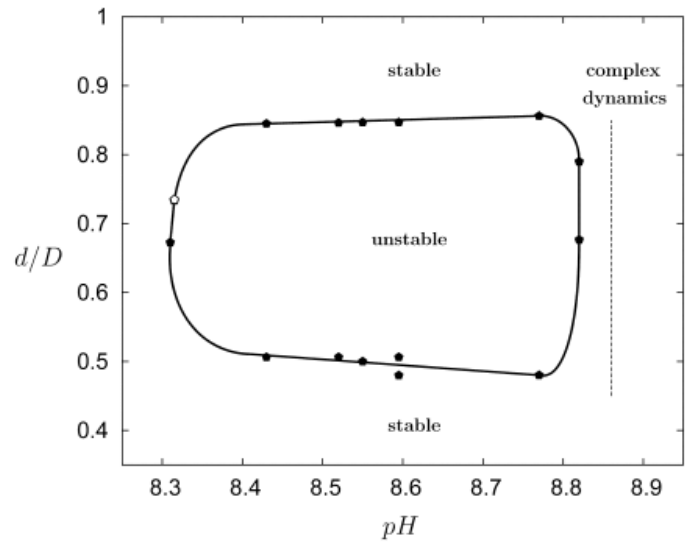

Fig. 7. Stability diagram for the longitudinal oscillations observed at high $\mathrm{pH}$. The open symbol on the left exhibited is both longitudinal and transverse oscillations. The vertical line marks a region of complex sphere dynamics

\subsection{Transverse Oscillations}

While taking measurements at $\mathrm{d} / \mathrm{D}=0.7339$ for $\mathrm{pH}=$ 8.315 corresponding to the open symbol on the left in Fig. 7, we observed two types of oscillations: a high frequency transverse (sideways) oscillation soon after release of the sphere that persisted to about mid-height, with recovery back to the low-frequency longitudinal oscillation over the lower half of the tube. The recovery back to longitudinal oscillation seems to have been initiated by a disturbance caused at the joint where two tubes are fused together. The transverse oscillations were accompanied by loud (one could say violent) pinging sounds as the sphere collided with the inner wall of the tube. Taking measurements with the other spheres we find the results given in Fig. 8. For $0.7339 \leq$ $\mathrm{d} / \mathrm{D} \leq 0.8375$ transverse oscillations are observed while for $0.6727 \leq \mathrm{d} / \mathrm{D} \leq 0.7339$ longitudinal oscillations are found. At the transition value $\mathrm{d} / \mathrm{D}=0.7339$ each type of oscillation is observed in separate parts of the tube. The variation of the measured frequencies for the longitudinal and transverse oscillations as a function of d/D are plotted in Fig. 9 for another mixture at $\mathrm{pH}=$ 8.31. Note that the curves are continuous in value, but discontinuous in slope.

\subsection{Complex dynamics}

We now describe the observations made along the vertical line at $\mathrm{pH}=8.86$ shown in Fig. 7 , well above the upper limit for pure longitudinal oscillations. It is to be noted that at $\mathrm{pH}=8.82$, and especially at $\mathrm{pH}=8.86$, the HPG mixture was indeed a fully-connected gel we had difficulties pouring the solution into the 2000 $\mathrm{ml}$ beaker which required us to squeeze and pinch off the mixture by hand.

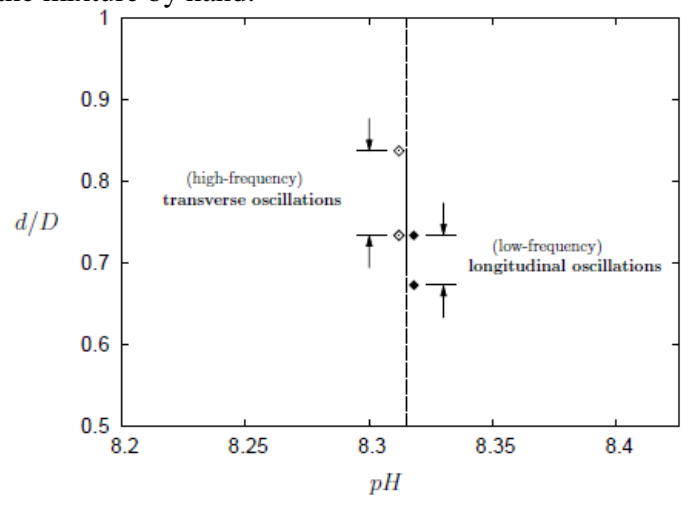

Fig. 8. Observation of both transverse and longitudinal oscillations at $\mathrm{pH}=8.31$ 


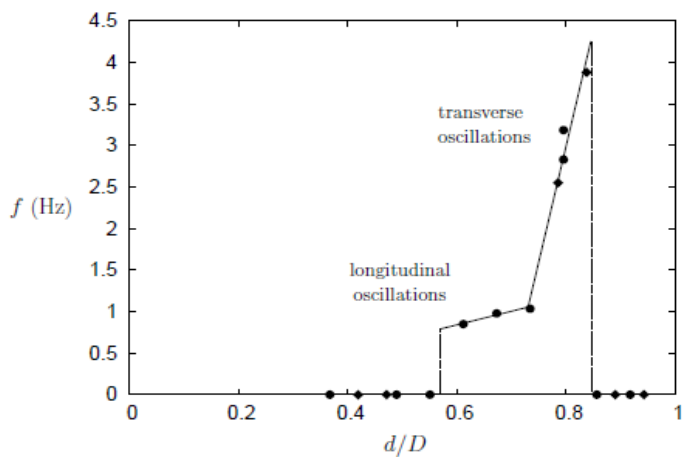

Fig. 9. Measurements of the frequencies of transverse and longitudinal oscillations at $\mathrm{pH}=8.31$. Circles are data from tube $D_{1}$ and triangles are data from tube $D_{2}$

At this highest $\mathrm{pH}$, complex dynamics were observed. As depicted in the frequency plot in Fig. 10, complicated roll-slip oscillations sporadically interspersed between the longitudinal oscillations were apparent.

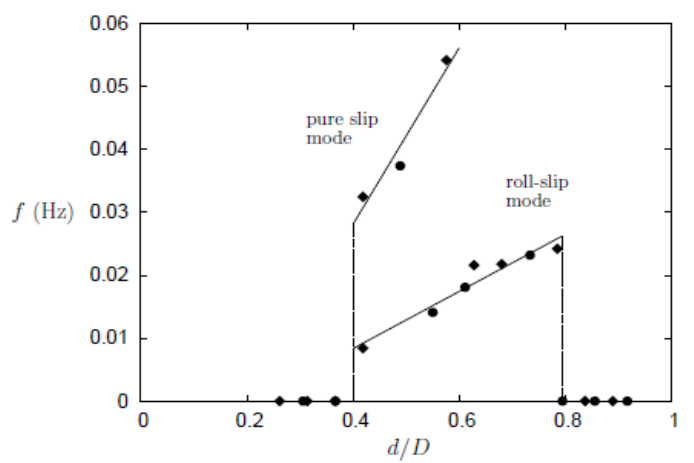

Fig. 10. Comparison of frequencies for the interspersed longitudinal and roll-slip modes of oscillation observed at $\mathrm{pH}=8.86$. Circles are data from tube $D_{1}$ and triangles are data from tube $D_{2}$

The kinematics of the motion of these modes is depicted in Fig. 11. The pure longitudinal mode in Fig. 11(a) has the sphere falling down at unsteady velocity $\mathrm{V}$ slipping past the vertical cylinder wall. For the roll-slip mode, the sphere would slip for a period of time without rotation and then would simultaneously slip and roll with angular speed $\Omega$ as depicted in Fig. 11(b), with the sense of rotation being up the cylinder wall. Such anomalous (uphill) rotation of spheres sliding down steeply inclined surfaces in other viscoelastic fluids has been observed and analyzed previously; see Joseph, et al. (1992), Liu, et al. (1993) and Seddon \& Mullin (2006). The results plotted in Fig. 10 show that the frequency of the roll-slip mode is about $1 / 3$ that of the longitudinal mode at each value of $\mathrm{d} / \mathrm{D}$.

\section{TransVerse AND HeliCAL OSCILLATIONS}

We next tracked the stability boundary for the highfrequency transverse oscillation mode. A small but discernible precession of the transverse oscillations was observed at high $d D$, as detected by two observers viewing the motion on orthogonal sides of the vertical tube.

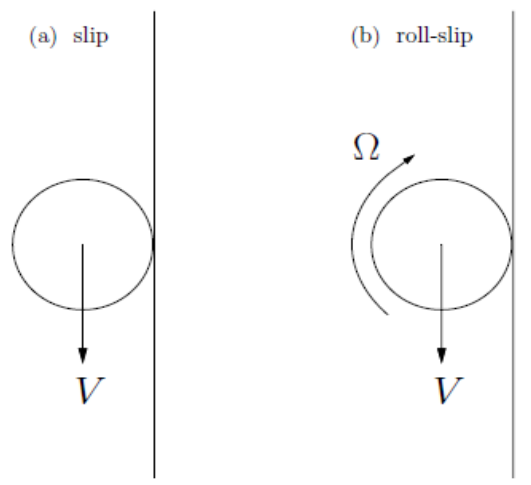

Fig. 11. Schematic of the slip and roll-slip behavior observed at $\mathrm{pH}=8$.

The importance of this feature will be considered further in $\$ 6$. This mode of oscillation persisted down to approximately $\mathrm{pH}=8.15$ at which point the spheres executed helical motions at both low and high values of $\mathrm{d} / \mathrm{D}$ as shown in Fig. 12. The upper transverse mode stability boundary is seen to cleanly bifurcate into the tight helix motion while the lower transverse mode stability boundary executes a curious $\mathrm{S}$-shape wiggle to the wide helix motion. The vertical line at $\mathrm{pH}=8.0$ shows five transitions separating six distinct flow regimes: from bottom to top these are stable, wide helix, stable, transverse oscillation, tight helix and stable flows.

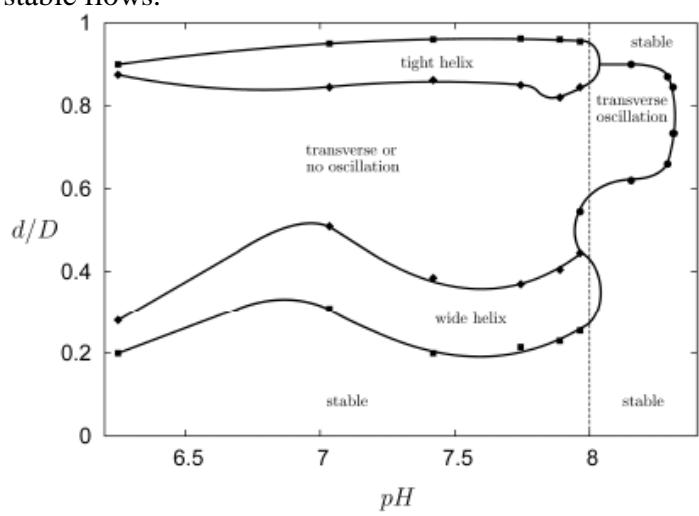

Fig. 12. Stability boundary for the transverse oscillations and helical motions observed at low $\mathrm{pH}$

At low values of $d / D$ the spheres executed a relatively low-frequency, wide spiraling motion during their descent; at most one could observe three helical turns over the height of the tube. At large values of $d / D$, on the other hand, the spheres exhibited a high-frequency, tight spiraling motion. Without the black circumscribed circles on the spheres, observation of this helical instability at high d/D from the front of the tube appears to the casual observer like a transverse instability. Its helical behavior was documented in two ways. First, the tight helix could be observed from above looking down along the tube. Second, the scribed circles revealed a slow spin during its descending helical trajectory; typically the sphere would spin one revolution about its vertical axis for each 6-10 revolutions of its tight helical motion. 
Sandwiched between the wide helix behavior at low $\mathrm{d} / \mathrm{D}$ and the tight helix motion at large $\mathrm{d} / \mathrm{D}$ are either transverse oscillations or straight drops void of any discernible instability. No clear boundaries between these motions were apparent. The three regions - wide helix, transverse or no oscillation, and tight helix could be tracked down to our lowest value $\mathrm{pH}=6.25$ which represents data for the HPG with no titration. As noted in Power, et al. (1998) the untitrated HGP is essentially a Newtonian fluid. At the far left boundary we did sometimes observe transverse oscillations, but they generally damped out during their fall down the tube. This suggests that a Newtonian fluid cannot support transverse oscillations of a sphere. However, the rocking motion of falling spheres with slight transverse oscillations has been documented by Viets \& Lee (1971), but this is due to the center of mass of the sphere being displaced from it geometric center.

\section{DisCuSSION AND CONCLUSION}

Our current understanding of the dynamics of spheres falling through HPG contained in tall glass tubes is limited to $\mathrm{pH}$ values in the range $6.25<\mathrm{pH}<8.86$, the results of which are summarized in Fig. 13.

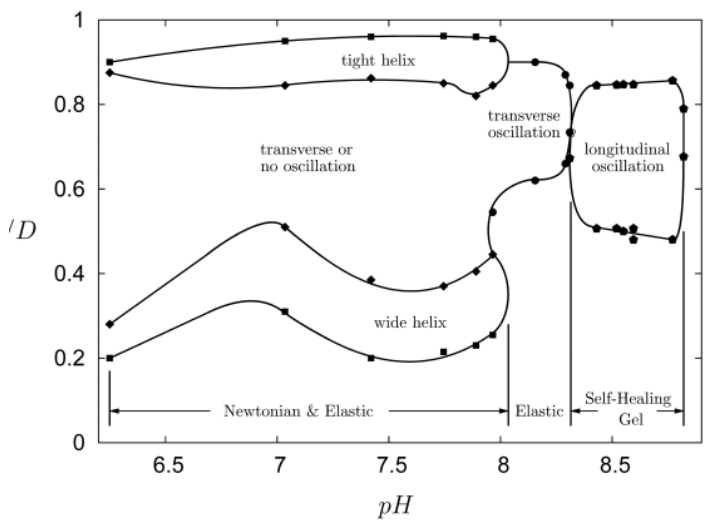

Fig. 13. Composite stability diagram for the longitudinal, transverse and helical modes

The boundary for the transverse oscillations is slightly oblique to the boundary for longitudinal oscillations, with a kissing point at $\mathrm{pH}=8.315$. As depicted in Fig. 13 it is apparent that the self-healing nature of the gel is responsible for the longitudinal oscillations in the region $8.31<\mathrm{pH}<8.82$. The upper limit of the longitudinal oscillations shown most clearly in Fig. 7 is near $\mathrm{pH}=8.82$. We also have noted that at this value of $\mathrm{pH}$ our guar behaved like a fully connected gel; at the lower value $\mathrm{pH}=8.77$ the gel was slightly more fluidlike than solid-like. Apparently the transition point for our gel lies in the range $8.77<\mathrm{pH}<8.82$ close to the value $\mathrm{pH}=8.7$ found by Power, et al. (1998). We conclude that the upper limit of the stability boundary for longitudinal oscillations is very near the HPG gel point.

In the region $8.035<\mathrm{pH}<8.31$ only transverse oscillations appear in a limited range of $\mathrm{d} / \mathrm{D}$ and this is denoted the Elastic regime in Fig. 13. However, the existence of sporadic transverse oscillations below $\mathrm{pH}$
$=8.035$ suggests that the guar retains its elasticity down to low $\mathrm{pH}$, but must disappear and become completely Newtonian when the HPG is not tritated at the lowest value $\mathrm{pH}=6.25$. Thus we have labeled the region 6.25 $<\mathrm{pH}<8.035$ as Newtonian \& Elastic.

We speculate a little more on the physics of the various instabilities found here. For the longitudinal oscillations, Mollinger, et al. (1999) visualized the flow behind the sphere using small aluminum flakes. They reported that the general flow pattern during the deceleration stage is that of a wake dragging fluid along behind the sphere and the wake terminates in a stagnant region high (10-15 d) above the sphere. At instants when the sphere suddenly jumps, it appeared as if the wake acted like a breaking rubber band, enabling the sphere to accelerate rapidly.

Our interpretation, not in conflict with that given above, is that the time constant associated with the longitudinal oscillations is related to the healing time of the gel; at high Reynolds numbers the sphere can exert sufficient shear to break the gel-like nature of the fluid allowing the sphere to fall more rapidly, but the gel then selfheals and the sphere slows down. In our mixture, longitudinal oscillations occur only in the range $0.48<\mathrm{d} / \mathrm{D}<0.86$. For Reynolds numbers below a critical value, there is insufficient shearing to disrupt the gel structure and no longitudinal oscillations are observed; this evidently happens for $\mathrm{d} / \mathrm{D}<0.48$ owing to the small sphere diameters, and for $\mathrm{d} / \mathrm{D}>0.86$ when the settling speed is greatly reduced by blockage effects. We note from Fig. 1 that the minimum value for longitudinal oscillations reported by Mollinger, et al. (1999) is $\mathrm{d} / \mathrm{D}=0.2$, well below our minimum value $\mathrm{d} / \mathrm{D}=0.48$. But one must bear in mind two things. First, the HPG used by Mollinger, et al. (1999) was prepared from Halliburton powder and ours from Clearwater powder. Probably the most important distinction to be made is that Mollinger, et al. (1999) was able to drop their spheres along the center of their tubes, while ours generally slipped down the inner tube wall.

Above the limit $\mathrm{pH}=8.82$ for longitudinal oscillations, there appears at $\mathrm{pH}=8.86$ a roll-slip mode interspersed between the longitudinal oscillations, the former having frequencies about $1 / 3$ the latter. The reduced frequency of the roll-slip mode is evidently due to the fact that the fluid is not so strongly sheared as the sphere attempts to roll up the cylinder wall while settling; cf. numerical simulations in Joseph, et al. (1992). It should be noted that the transition to a gel indicated by the value $\tan (\delta)=0$ in Fig. 2 occurs at $\mathrm{pH}=8.7$ (Power, et al. 1998). This is lower than the upper boundary $\mathrm{pH}=8.82$ of our longitudinal oscillations. If our HPG mixture is identical to that of Power, et al. (1998), then we are above the gel limit, yet the spheres still readily descend through the mixture. It is nearly certain that our mixture is not exactly that prepared by Power, et al. (1998), though we followed their mixture procedure very closely; if the mixtures are not exactly the same, then their gelation points will not be identical. Moreover, the definition of the gel point is simply a mathematical abstraction; heavy spheres may yet easily penetrate an HPG solution above its gel point.

The transverse oscillations that exist below $\mathrm{pH}=8.31$ perhaps owe their existence to the fluid's 
viscoelasticity. When a sphere is not captured by the tube wall, it is free to oscillate against the elastic forces in the confined fluid. Moreover, the curved cylinder walls evidently focus and trap the oscillation in a vertical plane cutting through the axis of the cylinder. In some instances, especially at high $\mathrm{d} / \mathrm{D}$, a precession of the plane of transverse oscillations was observed. This allows for an alternative physical explanation for the transverse oscillations at high $\mathrm{d} / \mathrm{D}$. Perhaps these high-frequency transverse oscillations are really the result of an asymmetrically shed wake which, if not confined by the vertical tube wall, would result in spiral motion; in this case the tube intercepts the spiral and the result is a transverse oscillation whose plane of oscillation slowly processes.

It is of interest to understand, for $6.25<\mathrm{pH}<8$, when one might expect to observe helical or transverse oscillations depending on the value of $d / D$. The competition between these modes of instability is probably tied to the Reynolds number of the settling sphere. For the highest descent Reynolds numbers which occur when $0.6<\mathrm{d} / \mathrm{D}<0.8$, unconfined transverse oscillations are sometimes observed; at lower and higher values of $d / D$ the Reynolds numbers decrease significantly and helical modes are observed. At the lower Reynolds numbers the shedding frequency of spiral vortices, as measured by the Strouhal number, is small and this may allow sufficient time for the impulse torque imparted to the sphere to dominate over transverse viscoelastic forces to produce the helical trajectories. This is a possible scenario for Reynolds numbers small by virtue of their diameter when $d / D$ is small.

We now discuss the motion observed at $\mathrm{pH}=6.25$ for the untitrated HPG which is Newtonian. Small windows of tight helical motion at high $\mathrm{d} / \mathrm{D}$ and wide helical motion at low d/D are observed in Fig. 13. This infers that the helical motion of spheres falling through tubes, or perhaps in unbounded domains, should be observed in Newtonian fluids in the absence of HGP powder. Indeed, helical descents have been observed in Newtonian fluids and this is attributed to the rotation of the wake which eventually induces the sphere to follow a spiral trajectory; see Viets (1971), Achenbach (1974) and Clift, et al. (1978). Direct measurement of the spiral motion of falling spheres has been documented by Uhlherr, Sinclair and Baxter (1965) as an example of their multiple image photographic technique for recording the path and velocity of moving objects. There are no details of the sphere diameters used in their experiment, nor of their densities, nor of the fluid used, which presumably was water for ease of photography. But the tube surrounding the falling spheres was glass with a diameter $7.6 \mathrm{~cm}$ and since the entire domain across the tube is visible in the time lapse images, we can estimate the diameter of the spheres shown in their Fig. 2. For the smaller sphere exhibiting spiraling motion in their Fig. 2(a) we find $d=2.0 \mathrm{~cm}$ corresponding to $d / D=0.27$. For the larger sphere exhibiting no spiraling motion in their Fig. 2(b) we find $\mathrm{d}=4.5 \mathrm{~cm}$ corresponding to $\mathrm{d} / \mathrm{D}=0.59$. Both of these values agree with our results at $\mathrm{pH}=6.25$ in Fig. 13 . The helical motion observed in our experiment at high $\mathrm{d} / \mathrm{D}$ at $\mathrm{pH}=6.25$ is also most likely due to the spiral vortex shed behind sedimenting spheres. To investigate, experiments were performed by PDW while visiting IRPHE, University of Provence in Marseille, France in 2001 The motion of glass marbles falling through a tall plexiglass tube filled with distilled water were observed at $\mathrm{d} / \mathrm{D}=0.85$; the glass spheres fell in a tight helical trajectory down the tube with frequencies similar to those observed in the HPG system. We note that this value of $d / D$ is only slightly lower than the range 0.875 $<\mathrm{d} / \mathrm{D}<0.900$ for the existence of tight helical motion for the untitrated HPG mixture at $\mathrm{pH}=6.25$ shown in Fig. 13 .

In closing, we note that just after filling the tall tubes with an HPG solution, relatively large bubbles would dislodge themselves from being trapped under the ends of the tubes. In the region of longitudinal oscillations we observed the oscillation of those bubbles while rising up the tube. During the accelerating phase the cusped tail would grow in length and during the decelerating phase the cusped tail would shrink in length. These results are very similar to those observed by Belmonte (2000) in his study of bubbles rising through a micellar solution.

\section{ACKNOWLEDGEMENTS}

The authors are grateful to Clearwater, Inc., 5605 Grand Ave., Pittsburg, PA 15225, USA for donating the WGA-1 hydroxypropoguar gelling agent used in this experiment. We are especially indebted to Alex Mollinger (Shell International Exploration and Production in Rijswijk, The Netherlands) for informative discussions on aspects of this experiment and inherent difficulties in the preparation of the guar. Our university glassblower Dennis Steffey fused together the tall tubes used in the experiment. PDW extends his thanks to Christophe Clanet for his hospitality while visiting IRPHE in Marseilles and for allowing use of his laboratory facilities to conduct the experiment for glass marbles falling through water. The authors acknowledge Joseph Kubitschek and Michael Sprague for assistance with some of the figures.

\section{REFERENCES}

Achenbach, E. (1974). Vortex shedding from spheres, J. Fluid Mech., 62, 209-221.

Barnes, H. A., J. F. Hutton and K. Walters (1989). An Introduction to Rehology, Elsevier, New York.

Belmonte, A. (2000). Self-oscillations of a cusped bubble rising through a micellar solution, Rehol. Acta, 39, 554-559.

Carnali, J. O. (1992). Gelation in physically associating biopolymer systems, Rheol. Acta, 31, 399-412.

Chen, S. and J. P. Rothstein (2004). Flow of a wormlike micelle solution past a falling sphere, J. NonNewtonian Fluid Mech., 116, 205-234.

Clift, R., J. R. Grace and M. E. Weber (1978). Bubbles, Drops and Particles, Academic Press, New York. 
Dhahir, S. A. and K. Walters (1989). On Newtonian flow past a cylinder in a confined flow, J. Rheol., 33, 781-804.

Eisen, S., B. Roberts and P. D. Weidman (2002). Three instability modes for spheres settling through a vertical pipe containing HPG, APS Division of Fluid Dynamics, 55th Annual Meeting, Dallas, TX, November 24-26.

Handzy, N. Z. and A. Belmonte (2004). Oscillatory rise of bubbles in wormlike micellar fluids with different microstructures, Phys. Rev. Lett, 92, 124501 .

Jayaraman, A. and A. Belmonte (2003). Oscillations of a solid sphere falling through a wormlike micellar fluid, Phys. Rev. E., 67, 065311(R).

Joseph, D. D., J. Nelson, H. Hu and Y. J. Liu (1992). Competition between inertial pressures and normal stresses in teh flow induced anisotropy of solid particles, P. Moldenaers and R. Keunings (Eds.), Theoretical and Applied Rheology, Elsevier, Amsterdam, pp. 60-65.

Lapasin, R. and S. Pricl (1995). Rheology of Industrial Polysaccharides: Theory and Applications, Chapman and Hall, London.

Liu, Y. J., J. Nelson, J. Feng and D. D. Joseph (1993). Anomalous rolling of spheres down an inclined plane, J. Non-Newtonian Fluid Mech., 50, 305330.

Mollinger, A. M., E. C. Cornelissen and B. H. A. A. van den Brule (1999). An unexpected phenomenon observed in particle settling: oscillating falling spheres, J. Non-Newtonian Fluid Mech., 86, 389393.

Power, D. J., A. B. Rodd , L. Paterson and D. V. Boger (1998). Gel transition studies on nonideal polymer networks using small amplitude oscillatory rheometry, J. Rheol., 42, 1021-1037.

Seddon, J. R. T. and T. Mullin (2006). Reverse rotation of a cylinder near a wall, Phys. Fluids, 18, 041703.

Roberts, B., S. Eisen and P. D. Weidman (2000). Oscillations of a sphere settling through a nonNewtonian fluid, APS Division of Fluid Dynamics, 53rd Annual Meeting, Washington, DC, November 19-21.

Uhlherr, P. H. T., C. G. Sinclair and B. A. A. Baxter (1965). A multiple image photographic technique for recording the path and velocity of moving objects, Chem. Engr. Sci., 20, 997-1000.

Viets, H. (1971). Accelerating sphere-wake interaction, AIAA Journal, 9, 2087-2089.

Viets, H. and D. A. Lee (1971). Motion of freely falling spheres at moderate Reynolds numbers, AIAA Journal, 9, 2038-2042.
Weidman, P. D., B. Roberts and S. Eisen (2002). Longitudinal, transverse and spiral instability modes of a sphere settling through a pipe filled with HPG, Progress in Nonlinear Science, International Conference decidated to the 100th Anniversary of A. A. Andronov, Volume III: Nonlinear Oscillations, Control and Information, Nizhny Novgorod, Russia, 103-114. 